\title{
ArcR modulates biofilm formation in the dental plaque colonizer Streptococcus gordonii
}

\author{
J.C. Robinson \\ N.S. Jakubovics ${ }^{1}$
}

${ }^{1}$ School of Dental Sciences, Newcastle University, Newcastle upon Tyne, UK

${ }^{2}$ Bioinformatics Support Unit, Newcastle University, Newcastle upon Tyne, UK

${ }^{3}$ Centre for Bacterial Cell Biology, Newcastle University, Newcastle upon Tyne, UK

${ }^{4}$ Department of Epidemiology, School of Public Health, University of Michigan, Ann Arbor, MI, USA

Correspondence

Nicholas S. Jakubovics, Centre for Oral Health Research, School of Dental Sciences, Newcastle University, Framlington Place, Newcastle upon Tyne, UK.

Email: nick.jakubovics@ncl.ac.uk

Funding information

Jeffcock and Luccock Newcastle University PhD studentship awarded to JCR.

\begin{abstract}
Summary
Biofilm formation and cell-cell sensing by the pioneer dental plaque colonizer Streptococcus gordonii are dependent upon arginine. This study aimed to identify genetic factors linking arginine-dependent responses and biofilm formation in S. gordonii. Isogenic mutants disrupted in genes required for the biosynthesis or catabolism of arginine, or for arginine-dependent gene regulation, were screened for their ability to form biofilms in a static culture model. Biofilm formation by a knockout mutant of $\operatorname{arcR}$, encoding an arginine-dependent regulator of transcription, was reduced to $<50 \%$ that of the wild-type whereas other strains were unaffected. Complementation of S. gordonii $\triangle \operatorname{arcR}$ with a plasmid-borne copy of $\operatorname{arcR}$ restored the ability to develop biofilms. By DNA microarray analysis, 25 genes were differentially regulated in S. gordonii $\triangle \operatorname{arcR}$ compared with wild-type under arginine-replete conditions including eight genes encoding components of phosphotransferase systems for sugar uptake. By contrast, disruption of $\operatorname{argR}$ or $\operatorname{ahrC}$ genes, which encode paralogous arginine-dependent regulators, each resulted in significant changes in the expression of more than 100 genes. Disruption of a gene encoding a putative extracellular protein that was strongly regulated in S. gordonii $\triangle \operatorname{arcR}$ had a minor impact on biofilm formation. We hypothesize that genes regulated by ArcR form a critical pathway linking arginine sensing to biofilm formation in S. gordonii. Further elucidation of this pathway may provide new targets for the control of dental plaque formation by inhibiting biofilm formation by a key pioneer colonizer of tooth surfaces.
\end{abstract}

\section{KEYWORDS}

ArcR regulon, dental plaque, microarray, oral streptococci, saliva

\section{1 | INTRODUCTION}

The formation of dental plaque is initiated by the attachment of pioneer colonizers to the tooth surface. ${ }^{1}$ Oral streptococci including Streptococcus gordonii, Streptococcus sanguinis, Streptococcus oralis and Streptococcus mitis are particularly well adapted for the initial colonization of tooth surfaces because they produce a multitude of cell surface adhesin proteins and glycoproteins that recognize host receptors in the salivary pellicle. ${ }^{2-4}$ Many of these adhesins also contribute to the subsequent development of dental plaque by mediating cell-cell binding, known as coaggregation, with other oral bacteria. ${ }^{5-7}$

Oral streptococci are considered to be opportunistic pathogens because they can enter the bloodstream and are among the leading causes of the rare, but life-threatening, disease infective endocarditis. ${ }^{8}$ However, in mature dental plaque there is evidence that oral streptococci protect against dental caries. Some species produce arginine deiminase, which generates ammonia and neutralizes plaque acid, leading to shifts in the microbiome towards health. ${ }^{9-11}$ Arginine deiminase also directly influences 
other oral bacteria by acting as an interspecies signaling molecule. For example, the arginine deiminase of Streptococcus cristatus is sensed by the periodontal pathobiont Porphyromonas gingivalis and leads to downregulation of virulence gene expression. ${ }^{12}$ The uptake of arginine by oral streptococci such as S. gordonii occurs through an arginine-ornithine antiporter, ArcD, and ornithine released into the growth medium can be used by other species such as Fusobacterium nucleatum. ${ }^{13}$ However, at high concentrations ( $\geq 50 \mathrm{~mm}$ ), arginine can disrupt coaggregation between $S$. gordonii and F. nucleatum by inhibiting the F. nucleatum adhesin RadD. ${ }^{14,15}$ High concentrations of arginine also disrupt multispecies oral microbial biofilms or S. gordonii monospecies biofilms. ${ }^{16-19}$

There is evidence that oral streptococci such as S. gordonii use arginine as a key signal for growth processes and biofilm formation. Streptococcus and related genera including Enterococcus, Lactococcus and Lactobacillus possess between two and four copies of ArgR family arginine-sensing transcriptional regulators, enabling close co-ordination of arginine biosynthesis, catabolism and transport. ${ }^{19}$ The $S$. gordonii genome, for example, encodes three different ArgR family regulators designated ArgR, AhrC and ArcR. Shifting S. gordonii cells from argininereplete medium to medium lacking arginine results in changes in expression of $>450$ genes, nearly one-quarter of the entire genome. ${ }^{19}$ Arginine sensing and biofilm formation pathways are triggered by intermicrobial interactions. For example, interactions with Candida albicans lead to downregulation of $S$. gordonii $\operatorname{arc} A$ and $\operatorname{arcB}$ encoding components of the arginine deiminase system, and upregulation of the biofilm-associated operon fruRBA. ${ }^{20}$ Coaggregation of S. gordonii with Actinomyces oris resulted in the co-ordinated downregulation of nine arginine biosynthesis genes and upregulation of the biofilm-promoting bfb locus. ${ }^{21}$ Coaggregation with A. oris also enabled the growth of $S$. gordonii in arginine-restricted medium. Therefore, it is possible that $S$. gordonii arginine-dependent regulators are employed to sense cell-cell interactions and respond by initiating growth or biofilm formation.

On the basis of the above observations, we hypothesized that one or more genes involved in arginine regulation and/or metabolism are linked to biofilm formation in oral streptococci. Streptococcus gordonii has been well-characterized in terms of responses to arginine, so we used this species as a model to explore the genetic basis of the link between arginine and biofilm formation by oral bacteria. Initially, a molecular genetic approach was employed to screen for components of arginine pathways (regulation, biosynthesis, or catabolism) that are linked to biofilm formation. To obtain further insights into argininemediated gene regulation, we characterized the regulons of the three S. gordonii ArgR homologues, ArcR, ArgR and AhrC.

\section{2 | MATERIALS AND METHODS}

\section{1 | Bacterial strains and growth conditions}

Bacterial strains used in this study are listed in Table S1. Streptococcus gordonii was routinely cultured in THYE medium containing $30 \mathrm{~g} \mathrm{~L}^{-1}$ Bacto $^{\mathrm{TM}}$ Todd Hewitt Broth (Becton Dickinson, Oxford, UK) and $5 \mathrm{~g} \mathrm{~L}^{-1}$ Yeast Extract (Melford Laboratories Ltd, Ipswich, UK) or on solidified THYE containing $15 \mathrm{~g} \mathrm{~L}^{-1}$ Bacto-agar (Becton Dickinson). Cells were cultured in candle jars without shaking for $24-48 \mathrm{~h}$ at $37^{\circ} \mathrm{C}$. For biofilm assays, S. gordonii was cultured in TYEG medium containing $10 \mathrm{~g} \mathrm{~L}^{-1}$ Bacto Tryptone, $5 \mathrm{~g} \mathrm{~L}^{-1}$ Yeast Extract, $3 \mathrm{~g} \mathrm{~L}^{-1} \mathrm{~K}_{2} \mathrm{HPO}_{4}$ and $2 \mathrm{~g} \mathrm{~L}^{-1} \mathrm{D}$-glucose, adjusted to $\mathrm{pH} 7.5$ before autoclaving. Escherichia coli was cultured in Luria-Bertani (LB) medium at $37^{\circ} \mathrm{C}, 250 \mathrm{rpm}$ or on LB medium solidified by the addition of $15 \mathrm{~g} \mathrm{~L}^{-1}$ Bacto-agar. When required, antibiotics were included in growth media at the following concentrations: erythromycin $2 \mu \mathrm{g} \mathrm{mL}^{-1}$, spectinomycin $100 \mu \mathrm{g} \mathrm{mL}^{-1}$, kanamycin $250 \mu \mathrm{g} \mathrm{mL}^{-1}$.

\subsection{Genetic manipulation of S. gordonii}

Routine genetic manipulations were conducted in accordance with standard protocols. ${ }^{22}$ Previously constructed gene knockout mutants are described in the Supplementary material (Table S1). Disruption of SGO_ RS04150 (here designated "earA") was performed using polymerase chain reaction (PCR) overlap extension mutagenesis as described by Jakubovics et al. ${ }^{19}$ Briefly, flanking regions of the earA gene were PCR amplified using primers earA F1 ovex and earA R1 kan ovex to generate an 869-bp product in the $5^{\prime}$ region of earA and earA F2 kan ovex and earA R2 ovex to generate a 903-bp product in the $3^{\prime}$ end of earA (see Table S2). The aphA3 cassette (910 bp) was amplified from plasmid pSF151 with primers kan F1 ovex/kan R1 ovex. The PCR products were stitched together in an overlap extension PCR. The resulting product was cleaned and used for transformation of S. gordonii DL1. Successful disruption and replacement of earA gene with the aphA3 cassette was confirmed by DNA sequencing.

To produce a genetic complementation strain (S. gordonii $\operatorname{arc} R_{\text {Comp }}$ ), plasmid parcR $R_{\text {Comp }}$ was generated using the In-Fusion HD PCR ligation cloning kit (Clontech, Mountain View, CA). A 181-bp region of the synthetic CP25 promoter was amplified from plasmid $\mathrm{pCM} 18,{ }^{23}$ using primers CP25F and CP25R. Primers pPE1010F and pPE1010R were used to amplify a 5652-bp fragment of vector pPE1010. ${ }^{24}$ Primers $\operatorname{arcRCompF}$ and $\operatorname{arcRCompR}$, containing 15-bp regions of overlap with pPE1010, were used to amplify a 494-bp fragment containing the $\operatorname{arc} R$ gene from S. gordonii chromosomal DNA. The In-Fusion HD PCR ligation cloning kit was employed to fuse the CP25 promoter and $\operatorname{arcR}$ gene into the pPE1010 vector. The integrity of plasmid parc $R_{\text {Comp }}$ was confirmed by sequencing, and parc $R_{\text {Comp }}$ was used for transformation of $S$. gordonii $\Delta \operatorname{arcR}:: a a d 9$ to generate $S$. gordonii $\operatorname{arcR} R_{\text {Comp }}$.

\section{3 | Crystal violet biofilm assay}

Biofilms for crystal violet assays were cultured on the surface of Cellstar ${ }^{\circledR}$ 96-well microtiter plates in TYEG medium without shaking, aerobically for $18 \mathrm{~h}$ at $37^{\circ} \mathrm{C}$ (Greiner Bio-one, Stonehouse, UK). The biomass was measured as described by Shields et al. ${ }^{25}$ Biofilms were submerged in $100 \mu \mathrm{L}$ of $0.5 \%(\mathrm{w} / \mathrm{v})$ crystal violet. After incubation at $20^{\circ} \mathrm{C}$ for $15 \mathrm{~min}$, wells were washed three times in $200 \mu \mathrm{L}$ of phosphate-buffered saline (PBS), air-dried, and residual crystal violet was dissolved with $100 \mu \mathrm{L}$ of $7 \%(\mathrm{v} / \mathrm{v}$ ) acetic acid and quantified by measuring $A_{562}$. All experiments were performed three times independently. Statistical significance of differences between mutants and wild-type $S$. gordonii was assessed by analysis of variance with Dunnett's post-hoc test, and $P<.05$ was considered significant. 


\subsection{Fluorescent staining and imaging}

Biofilms for visualization experiments were cultured on sterile glass coverslips incubated in wells of six-well tissue culture dishes. Following growth, biofilms were rinsed with PBS and incubated with Live/Dead BacLight stain (Molecular Probes, Eugene, OR) for $15 \mathrm{~min}$ at $20^{\circ} \mathrm{C}$. For confocal laser scanning microscopy, each stained coverslip was rinsed with PBS and inverted onto a PBS-filled Gene frame $(25 \mu \mathrm{L}, 1.0 \times 1.0 \mathrm{~cm}$, Thermo Fisher Scientific, Waltham, MA) secured on a microscope slide. Imaging was performed using a Nikon A1R confocal laser scanning microscope fitted with CFI PLAN APO VC objective (Nikon 60×/1.40 Oil). Images were captured with NIS-ELEMENTS C (v4.4, Nikon, Kingston upon Thames, UK) software and processed using IMARIS (v8.2, Bitplane, Zurich, Switzerland) software. Biovolume quantification of Z-stacks was conducted using VolociTy software (v6.3, PerkinElmer, Waltham, MA), set to identify objects $\geq 1 \mu \mathrm{m}^{2}$ as $S$. gordonii cells. At least three Z-stacks (image size $1024 \times 1024$ ) from three different fields of view were analyzed for each strain. The data were analyzed from three independent experiments. Statistical significance of differences between biofilm biovolume was assessed using analysis of variance with Tukey's post-hoc test.

\section{5 | Growth in chemically defined medium and RNA extraction}

For gene regulation analysis, chemically defined FMC medium ${ }^{19}$ was used with $0.5 \mathrm{mM}$ L-arginine $\mathrm{HCl}$ (Sigma-Aldrich, Dorset, UK) included or omitted as appropriate. Briefly, S. gordonii was cultured in FMC medium aerobically at $37^{\circ} \mathrm{C}$ for $18 \mathrm{~h}$. Cells were harvested, washed with fresh FMC and resuspended in $20 \mathrm{~mL}$ FMC medium. Cultures were incubated at $37^{\circ} \mathrm{C}$ until an optical density at $600 \mathrm{~nm}\left(\mathrm{OD}_{600}\right)$ of 0.3-0.4 was reached. At this point, cultures were split into $5-\mathrm{mL}$ aliquots, and cells were harvested and resuspended in $5 \mathrm{~mL}$ of either fresh arginine-replete FMC, or FMC without L-arginine. Cells were cultured at $37^{\circ} \mathrm{C}$ for a further $30 \mathrm{~min}$. To extract RNA, 1 volume of RNALater was added, cultures were vortex-mixed and incubated for $5 \mathrm{~min}$ at $20^{\circ} \mathrm{C}$. Cells were harvested and, after discarding the supernatant, pellets were stored for up to 5 days at $-80^{\circ} \mathrm{C}$. Cell pellets were thawed on ice and resuspended in $100 \mu \mathrm{L}$ spheroplasting buffer $\left(26 \%[\mathrm{w} / \mathrm{v}]\right.$ raffinose, $10 \mathrm{mM} \mathrm{MgCl}_{2}, 20 \mathrm{~mm}$ Tris- $\mathrm{HCl}$, $\mathrm{pH}$ 6.8) containing $0.1 \mathrm{mg} \mathrm{mL}^{-1}$ chloramphenicol or spectinomycin. Mutanolysin $\left(500 \mathrm{U} \mathrm{mL}^{-1}\right)$ was added to cells and incubated at $37^{\circ} \mathrm{C}$ for 5 min before addition of $350 \mu$ L RNAWiz solution (Thermo Fisher Scientific). The mixture was vortexed vigorously for $15 \mathrm{~s}$ and RNA was extracted using the Ambion RiboPure Bacteria RNA Purification kit (Thermo Fisher Scientific) in accordance with the manufacturer's recommendations.

\subsection{Gene expression analysis by quantitative reverse transcription PCR}

For quantitative reverse transcription PCR (RT-qPCR), samples were reverse transcribed with a QuantiTect Reverse Transcription kit (Qiagen, Valencia, CA). Reactions were performed according to the manufacturer's instructions, with the modification that $3 \mu \mathrm{g} \mathrm{m}^{-1}$ random hexamer primers (Bioline, London, UK) were used in place of the QuantiTect oligo-dT primers. The cDNA was cleaned and used as template in RT-qPCR experiments with the SensiMix SYBR No-ROX kit (Bioline) with the following reaction conditions: $1.95^{\circ} \mathrm{C}$ for $10 \mathrm{~min}, 2.95^{\circ} \mathrm{C}$ for $15 \mathrm{~s}, 3.60^{\circ} \mathrm{C}$ for $1 \mathrm{~min}$, 4. plate read, 5. repeat from step 2 a further 39 times, 6. melting curve from $55^{\circ} \mathrm{C}$ to $90^{\circ} \mathrm{C}$, read every $1^{\circ} \mathrm{C}$, hold for $5 \mathrm{~s}$. All samples were normalized against the $16 \mathrm{~S}$ rDNA gene. Primer sets for this and for $\arg C, \operatorname{argG}, \operatorname{pyr} A b, \operatorname{arc} A, \operatorname{arcB}, \operatorname{arc} D$ and $\operatorname{amy} B$ genes have previously been published. ${ }^{19}$ Other primers are described in Table S2. Standard curves, melting curves and agarose gel electrophoresis analysis of the cDNA were routinely included to validate the RT-qPCR experiments.

\section{7 | Microarray analysis}

Microarray analysis was performed as described by Jakubovics et al. ${ }^{19}$ using a previously designed microarray containing 2051 probes for $S$. gordonii genes (GEO accession GPL17786). Samples of RNA from four independent experiments per strain/growth condition were sent to the Genomics and Microarray Facility, Birmingham University, UK for reverse transcription, labeling and hybridization. The microarray series for S. gordonii DL1 in high or no arginine was previously deposited as GEO accession GSE51346. Other data series for arginine-dependent regulator mutants in high or no arginine were deposited in GEO under the accession number GSE101509.

Data were analyzed using Agilent GeneSpring GX software. Probe expression data were quantile normalized to enable unbiased comparisons between samples. To assess the relatedness of samples, principal component analysis (PCA) was carried out using the normalized data. Outliers identified in the PCA analysis were removed before proceeding to significance analysis. A moderated $t$-test was used to determine statistics for each probe. The resulting $P$-values were then adjusted using the Benjamini-Hochberg multiple testing correction procedure. ${ }^{26}$ For each comparison, probes with a corrected $P$-value of $\leq .05$ and with fold change numerically greater than 2 were considered to be differentially expressed between conditions. Rank product analysis was carried out using the RP method implemented in the R package "RankProd". ${ }^{27,28}$ This method applies a non-parametric statistical test, based on rank ordering of genes according to fold changes, to detect genes that are consistently upregulated or downregulated in replicated experiments. The heatmap was produced using the $\mathrm{R}$ package ComplexHeatmap. ${ }^{29}$

\section{3 | RESULTS}

\section{1 | Effects of disrupting arginine metabolism or regulation genes on $\mathrm{S}$. gordonii biofilm formation}

To investigate the genetic basis for the previously observed links between arginine sensing and biofilm formation in S. gordonii, a 
range of mutants lacking genes involved in arginine-dependent regulation, arginine catabolism or biosynthesis were screened for their ability to form biofilms in a high-throughput static 96well microplate system. Biofilms were cultured for $24 \mathrm{~h}$ in TYEG medium, washed and stained with crystal violet to quantify the biofilm biomass (Figure 1). Biofilm formation was not significantly different from wild-type levels in any of the single mutants screened with the exception of $S$. gordonii $\triangle \operatorname{arcR}$, which formed approximately $50 \%$ less biofilm than S. gordonii DL1. This was not due to a defect in planktonic growth as the growth yield, measured as the $O D_{600}$ of the well before washing and staining, was almost identical between S. gordonii DL1 and $\triangle \operatorname{arcR}$ (data not shown). It was consistently observed that biofilms formed by $S$. gordonii $\triangle \operatorname{arcR}$ appeared similar to those produced by the wild-type until they were agitated, indicating that cells were loosely attached. The $\operatorname{arcR}$ gene encodes an arginine-dependent regulator of the ArgR family. To assess whether ArcR acts in concert with other ArgRfamily regulators to control biofilm formation, the $\operatorname{arcR}$ mutation was introduced into $S$. gordonii $\triangle a r g R$ and $\triangle a h r C$ backgrounds. In each case, biofilm formation by the double mutants containing an $\operatorname{arcR}$ knockout was similar to that of the $\triangle \operatorname{arcR}$ single mutant, and approximately $50 \%$ reduced compared with wild-type. By contrast, an S. gordonii $\Delta \operatorname{argR} \triangle a h r C$ double mutant was not impaired in biofilm formation (Figure 1). Therefore, it appears that ArcR and not $\mathrm{ArgR}$ or $\mathrm{AhrC}$ is required for efficient biofilm formation in $S$. gordonii.

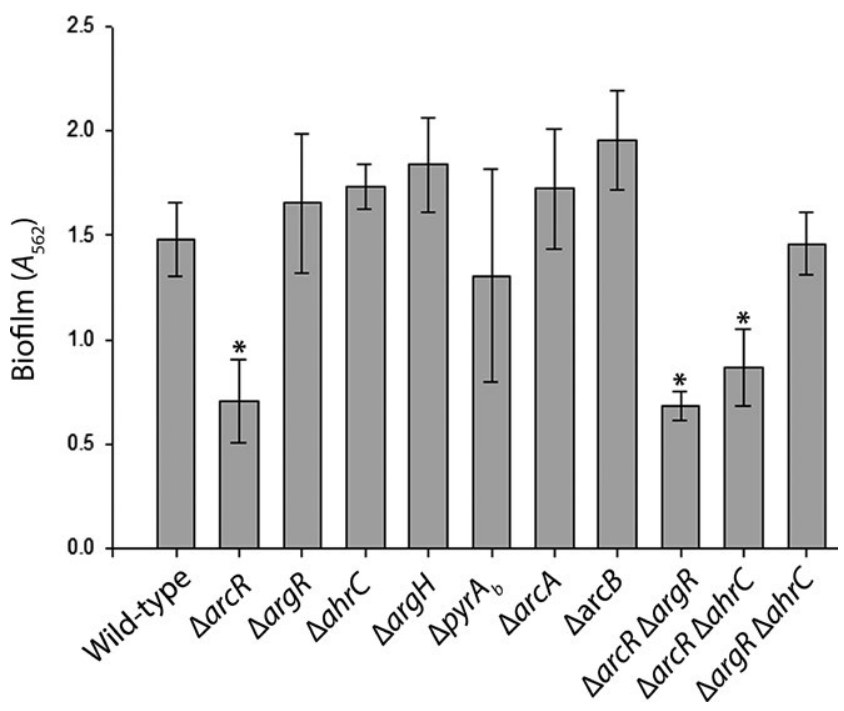

FIGURE 1 Biofilm formation by Streptococcus gordonii DL1 and isogenic mutants disrupted in arginine metabolism or regulation genes. Biofilms were grown aerobically in TYEG medium for $18 \mathrm{~h}$, and quantified by measuring absorbance of crystal violet-stained cells at $562 \mathrm{~nm}$. Biofilm formation by $\Delta$ arcR single- or double-mutant strains was significantly lower than those of the other mutant strains $(P<.05$; indicated by asterisks). Bars represent arithmetic means of three independent biological repeats, and standard error is shown. All S. gordonii $\Delta$ arcR mutant strains show a significant biofilm defective phenotype, not displayed by the other mutants tested here

\subsection{Visualization of biofilms and genetic complementation of $S$. gordonii $\Delta \operatorname{arcR}$}

Analysis of biofilms by confocal laser scanning microscopy with BacLight LIVE:DEAD staining revealed clear differences between biofilms formed by S. gordonii DL1 and S. gordonii $\triangle \operatorname{arcR}$ (Figure 2). Biofilms formed by the wild-type were confluent and relatively smooth, and approximately 10-20 $\mu \mathrm{m}$ thick throughout. By contrast, S. gordonii $\triangle \operatorname{arcR}$ biofilms were more heterogeneous, with clumps of cells up to $40 \mu \mathrm{m}$ thick and patches of surface that were not covered at all. To confirm that the observed biofilm defects were due to disruption of the $\operatorname{arcR}$ gene and not a second site mutation, the $\operatorname{arcR}$ gene was reintroduced into $S$. gordonii $\triangle \operatorname{arcR}$ on a plasmid under regulation of the synthetic CP25 promoter to generate $S$. gordonii $\operatorname{arc} R_{\text {Comp. }}$. Biofilms formed by $S$. gordonii $\operatorname{arc} R_{\text {Comp }}$ were similar in structure to those of the wild-type. Quantitative assessment using image analysis software demonstrated that the biovolume of $S$. gordonii $\Delta \operatorname{arcR}$ biofilms was significantly reduced compared with wild-type $S$. gordonii or the genetic complementation strain $S$. gordonii $\operatorname{arcR}_{\text {Comp }}$, indicating that the biofilm formation defect observed in S. gordonii $\triangle \operatorname{arcR}$ was a direct result of $\operatorname{arcR}$ gene disruption.

\subsection{Analysis of the ArcR regulon}

To assess the effects of disrupting the $\operatorname{arcR}$ gene on global gene expression in S. gordonii, microarrays were employed to analyze gene expression in S. gordonii DL1 or $\Delta \operatorname{arcR}$ in high arginine or following a shift to no arginine. Cells were cultured to mid-exponential phase in arginine-replete chemically defined growth medium, harvested and resuspended in high (0.5 mm) or no arginine. After $30 \mathrm{~min}$, RNA was extracted and gene expression was monitored by microarray. Initially, the results from the microarray were validated by assessing the expression levels of seven genes under high and no arginine using RT-qPCR. The genes $\arg C, \arg G, \operatorname{pyr} A b, \operatorname{arc} A, \operatorname{arcB}, \operatorname{arc} D$ and amyB (Figure 3) were selected for this analysis. Of these, amyB was included as a control, and the other genes were chosen because they were previously reported to be implicated in arginine metabolism and transport. ${ }^{19}$ The comparison showed a strong correlation between the microarray and RT-qPCR, with $r^{2}$ values of .995 and .777 for S. gordonii DL1 and $\Delta \operatorname{arcR}$, respectively, and slopes very close to 1 for each strain. To further assess the validity of individual microarray experiments, gene regulation from all S. gordonii DL1 and $\triangle \operatorname{arcR}$ microarray samples under high and no arginine was compared by principle coordinates analysis (PCoA; data not shown). Four independent experiments were performed for each strain under each condition, giving a total of 16 microarray samples. However, PCoA identified two outliers in the data: one sample each of S. gordonii DL1 under high arginine and no arginine. These samples were removed from subsequent data analysis.

In total, 26 genes were significantly different between $S$. gordonii DL1 and $\triangle \operatorname{arcR}$ under arginine-replete conditions (Table S3). This included arcR itself, which gave a detectable signal in the knockout strain even though the gene was not present. This signal was very low and 

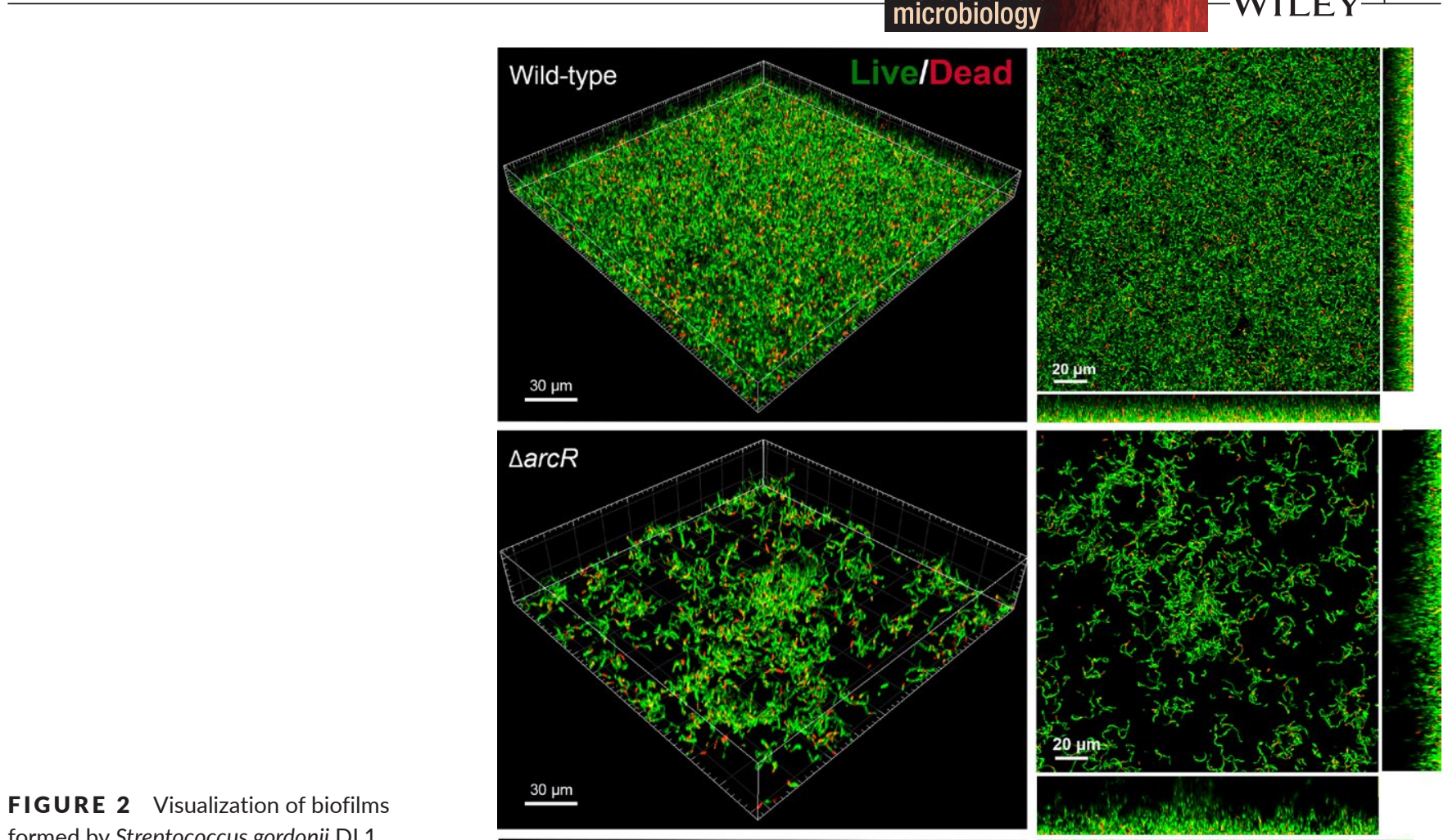

FIGURE 2 Visualization of biofilms formed by Streptococcus gordonii DL1, $\triangle \operatorname{arcR}$ and $\operatorname{arc} R_{\text {Comp. }}$. Biofilms were grown in TYEG medium aerobically for $18 \mathrm{~h}$, stained with BacLight LIVE:DEAD stain and visualized by confocal laser scanning microscopy. Three-dimensional renderings (left) or maximum projection images (right) are shown. Live cells stained with Syto 9 appear green; red staining shows compromised cells that have taken up propidium iodide. Images were quantified using Volocity software. The quantification (mean \pm standard errors) of biovolume is shown for three independent experiments. Biovolumes of biofilms from all strains were significantly different from one another

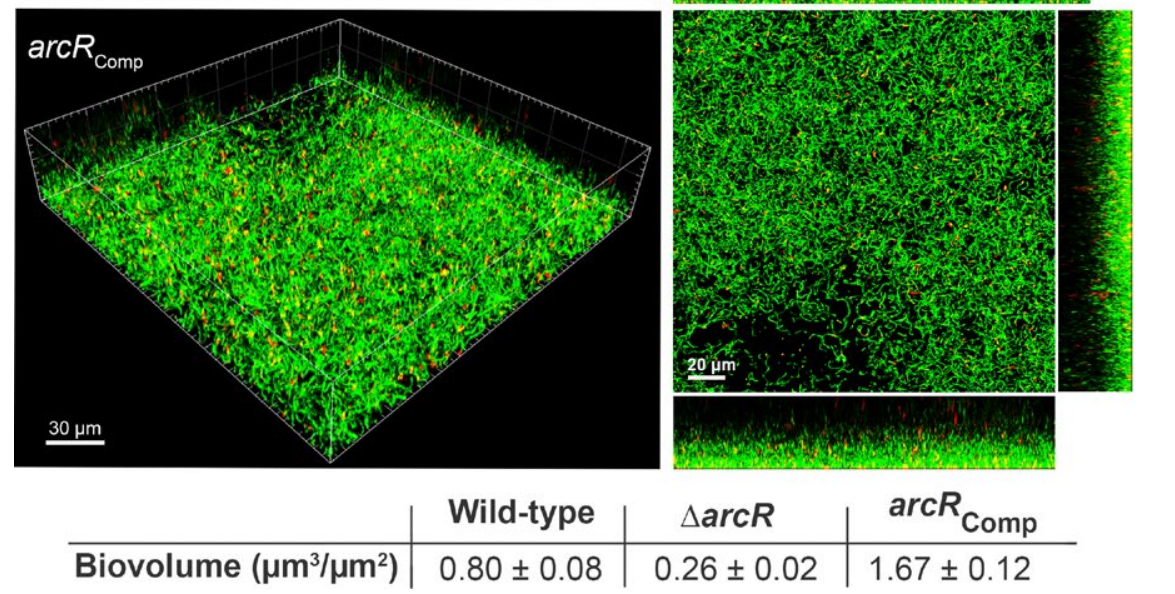

was not dependent on arginine, and therefore was presumably due to background fluorescence. Of the other 25 genes, the most strongly regulated was a putative extracellular protein encoded by SGO RS04150, which was upregulated 110-fold in S. gordonii $\Delta$ arcR compared with the wild-type. The argGH operon (SGO_RS00865-00870) was upregulated five- to six-fold. The gene queA, encoding a putative $S$-adenosylmethionine: tRNA ribosyltransferase-isomerase, was upregulated 12 -fold in S. gordonii $\Delta \operatorname{arcR}$. An apparent four-gene operon (SGO_RS07910-07925) encoding a predicted magnesium transporter, a CAAX amino protease, a methyltransferase and peptide chain release factor 3, was upregulated four- to six-fold. Two operons encoding phosphotransferase system (PTS) components were also upregulated: manLM encoding mannose-specific enzyme IIAB and IIC was upregulated $~ 2.5$-fold and levDEFG encoding a fructose PTS enzyme IIA, IIB, IIC and la was upregulated 7- to 12-fold. In addition, three hypothetical proteins, a possible pseudogene, an additional CAAX amino protease, peptidase S11, a putative ATP-dependent Clp protease ATP-binding protein, and a possible carboxylate-amine ligase were each upregulated two- to four-fold. Only three genes were downregulated in $S$. gordonii $\triangle$ arcR: SGO_RS02495 and SGO_RS08100 encoding putative PTS enzyme IIABC components (both downregulated $\sim 2$-fold) and the coiA gene encoding a putative gene involved in genetic competence, which was nine-fold downregulated.

To assess the impact of the $\operatorname{arcR}$ gene knockout on the ability of $S$. gordonii to respond to arginine, the ratio of gene expression following a shift to no arginine versus maintenance in high arginine for each of the above genes was calculated for S. gordonii DL1 and $\triangle \operatorname{arcR}$ (Figure 4). This analysis revealed several different patterns of gene regulation. The two PTS operons manLM and levDEFG along with the four-gene operon containing a putative magnesium transporter (SGO_ RS07910-07925) had little or no arginine-dependent regulation in S. gordonii DL1 but were more strongly downregulated in response to arginine restriction in S. gordonii $\triangle \operatorname{arcR}$. The reverse was seen with SGO_ RS07765 encoding peptidase S11 and coiA, which were more strongly 

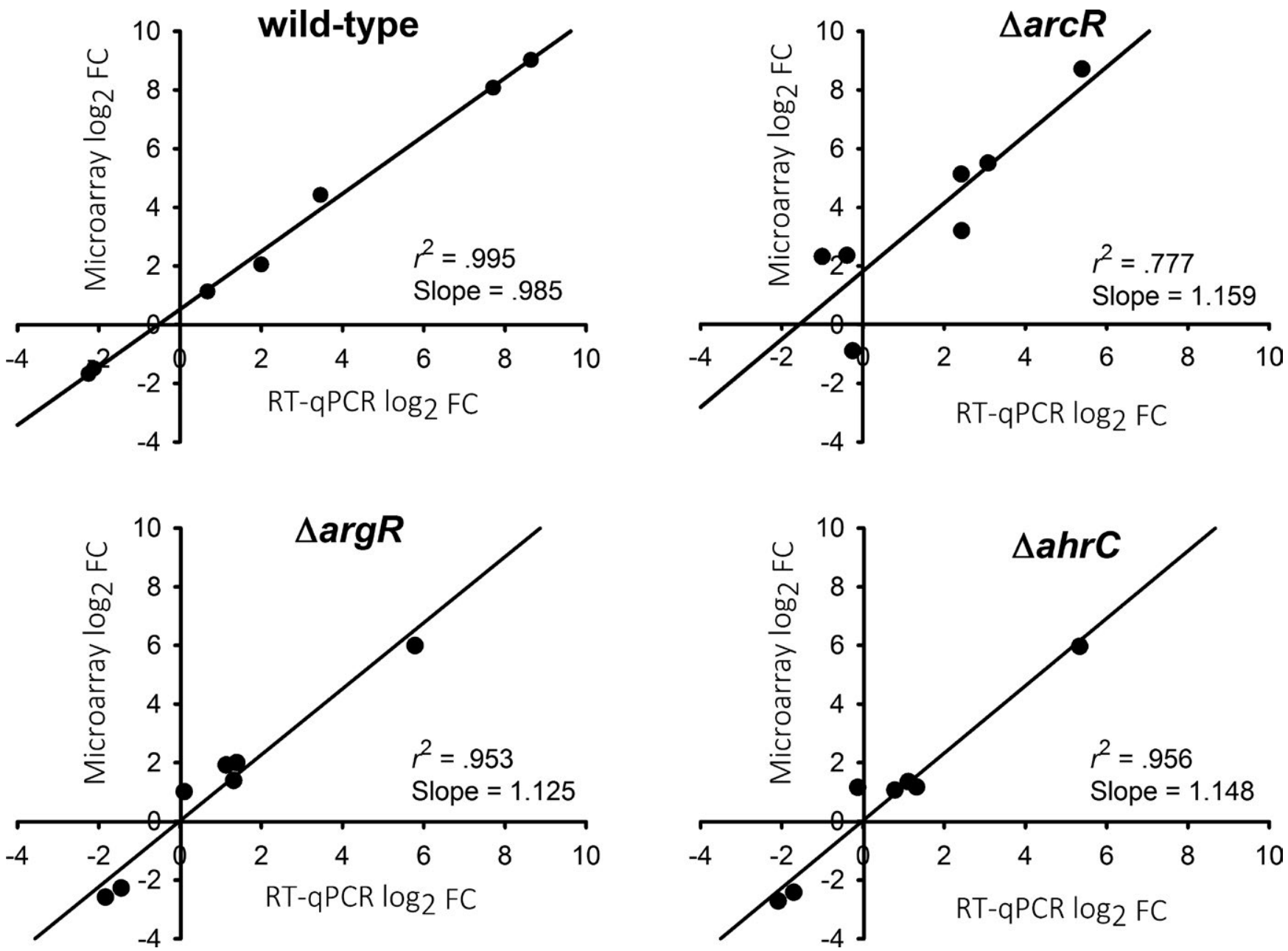

FIG URE 3 Validation of the Strpetococcus gordonii DL1, $\Delta \operatorname{arcR,} \Delta \operatorname{argR}$ and $\Delta a h r C$ microarrays by comparison with RT-qPCR data for each strain. Gene expression was measured as a $\log _{2}$ fold-change in no arginine compared with high arginine in $S$. gordonii DL1, $\Delta$ arcR, $\Delta a r g R$ and $\Delta a h r C$ strains. The linear regression line for each correlation is shown. Values on the graphs indicate the $r^{2}$ value and the slope of the line

downregulated in the wild-type than $S$. gordonii $\triangle \operatorname{arcR}$ in response to the removal of arginine. The queA gene, SGO_RS00730 (hypothetical protein) and SGO_RS09090 (Clp protease ATP binding protein) were upregulated in the absence of arginine to approximately the same extent in the wild-type and $\triangle \operatorname{arcR}$ mutant. SGO_RS02495 glucose PTS enzyme IIABC subunit, SGO_RS01240 encoding a CAAX amino protease, SGO_RS08100 encoding trehalose PTS enzyme IIABC and SGO_RS10240 carboxylate-amine ligase were expressed at similar levels in no versus high arginine in the wild-type, but were upregulated under arginine restriction in S. gordonii $\Delta$ arcR. Finally, SGO_RS07655 and SGO_RS04295 encoding hypothetical proteins, the pseudogene SGO_RS06275 and $\operatorname{argGH}$ were strongly downregulated under high arginine in the wild-type and less strongly downregulated in S. gordonii $\Delta \operatorname{arcR}$.

\subsection{Gene regulation in S. gordonii $\Delta \operatorname{argR}$ and $\Delta a h r C$}

To compare the ArcR regulon with those of the orthologous ArgRfamily regulators, ArgR and AhrC, microarray analysis was performed on the S. gordonii $\Delta \operatorname{argR}$ and $\Delta a h r C$ mutants. The validity of the microarrays was checked by RT-qPCR for the genes $\arg C$, $\operatorname{argG}$, pyrAb, $\operatorname{arcA}, \operatorname{arcB}, \operatorname{arcD}$ and $\operatorname{amyB}$ (Figure 3). As with the previous microarrays, there were strong correlations between the microarray and RT-qPCR data ( $r^{2}$ values > .95 and slopes close to 1 ). There were no significant differences between gene expression in S. gordonii $\triangle \operatorname{argR}$ or $\triangle a h r C$ under either high or no arginine. The microarrays for S. gordonii $\Delta \operatorname{argR}$ and $\Delta a h r C$ were performed by a different operator and at a different time from the S. gordonii DL1 and $\triangle \operatorname{arc} R$ arrays. Performing microarray analyses at different times can lead to "batch effects" where absolute gene expression levels are slightly different across arrays due to the use of different reagents or slight changes in protocol. ${ }^{30}$ Further, a relatively high proportion of probes $(\sim 19 \%)$ did not give a quantifiable signal from the S. gordonii $\Delta \operatorname{argR}$ and $\Delta a h r C$ samples. To assess the relationships between the microarrays, the data from each array were compared by PCoA (Figure S1). The data for S. gordonii $\Delta \operatorname{argR}$ and $\Delta a h r C$ clustered together and were well separated from S. gordonii DL1 and $\Delta \operatorname{arcR}$. Although it is possible that these observations reflect genuine biological differences between the strains, the comparisons between S. gordonii $\Delta \operatorname{argR/\Delta ahrC}$ and S. gordonii DL1/arcR must be 


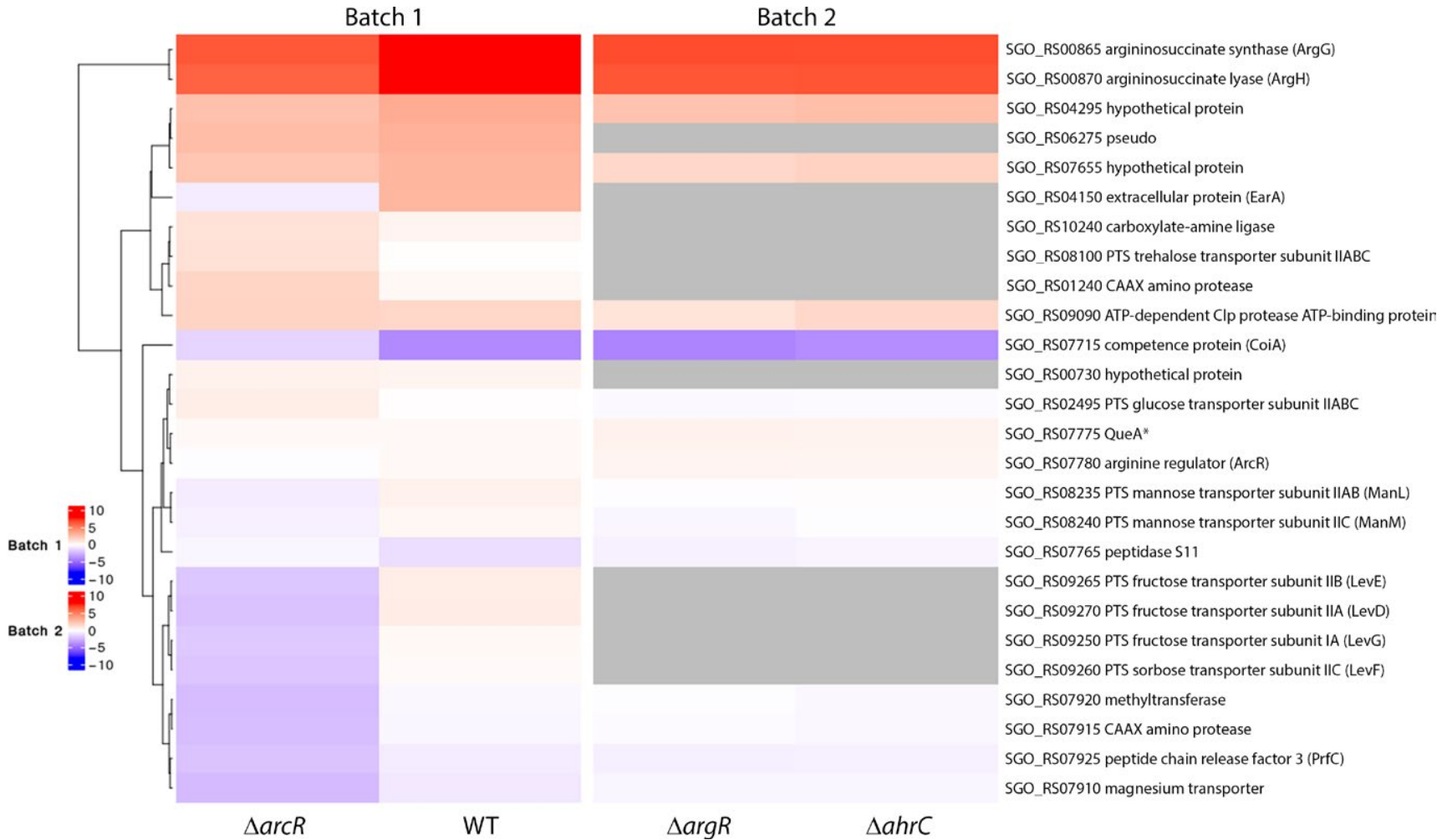

SGO_RS00865 argininosuccinate synthase (ArgG)

SGO_RS00870 argininosuccinate lyase (ArgH)

R04295 hypothetical protein

SGO_RS08100 PTS trehalose transporter subunit IIABC

GO RS01240 CAAX amino proteas

SGO RS08235 PTS mannose transporter subunit IIAB (ManL)

SGO_RS08240 PTS mannose transporter subunit IIC (ManM)

SGO_RS09270 PTS fructose transporter subunit IIA (LevD)

SCO RS09250 PTS fructose transporter subunit IA (LevG)

SGO_RS07925 peptide chain release factor 3 (PrfC)

WT

$\Delta \arg R$

$\Delta a h r C$

FIGURE 4 Heat map showing differential gene regulation in Streptococcus gordonii DL1, $\operatorname{arcR,\Delta } \arg R$ and $\Delta a h r C$ mutants in response to arginine. Microarray analysis was carried out on cDNA samples from S. gordonii strains during a shift to no arginine versus maintenance in high arginine. Color coding represents fold change in no arginine compared with high arginine, and negative values (blue tones) indicate downregulation. Data from S. gordonii DL1 and $\triangle \operatorname{arcR}$ strains are not directly comparable with those from S. gordonii $\Delta a r g R$ and $\Delta a h r C$ due to concerns about batch effects between the microarray experiments, which were conducted at different times. Gray areas in S. gordonii $\Delta a r g R$ and $\Delta a h r C$ microarrays show genes that did not pass the quality threshold, and for which no reading was obtained. *QueA is a predicted $S$-adenosylmethionine:tRNA ribosyltransferase-isomerase

treated with caution. Therefore, it was not possible to define the ArgR and AhrC regulons by direct comparison with S. gordonii DL1 under high or no arginine. Instead, rank product analysis was used to identify significant differences in gene expression between $S$. gordonii $\Delta \operatorname{argR} / \Delta a h r C$ and S. gordonii DL1 under high arginine.

Rank product analysis does not quantify the differences in expression levels between mutants and wild-type, but instead ranks the most strongly regulated genes. In total, 109 genes were identified using this approach that were differentially regulated in S. gordonii $\Delta \arg R$ and/ or $\Delta$ ahrC compared with S. gordonii DL1 (Table S4). The most strongly upregulated genes were the $\arg C J B D$ arginine biosynthesis operon, consistent with the previously identified roles of $\mathrm{ArgR}$ and $\mathrm{AhrC}$ as arginine-dependent repressors of transcription of arginine biosynthesis genes. Another arginine biosynthesis gene operon, $\operatorname{pyrRA} A_{a} A_{b}$, and the histidine biosynthesis operon (SGO_RS06875-SGO_RS06920) were also strongly and co-ordinately upregulated in the mutants. Approximately as many genes were downregulated in the mutants as upregulated. Downregulated genes included purE and purM, involved in purine metabolism, and scaC and scaA encoding components of an ABC-type manganese transporter.

Of the 26 genes identified as ArcR-regulated, 10 genes were not detected in the $S$. gordonii $\Delta \operatorname{argR}$ and $\Delta a h r C$ samples (shaded grey in Figure 4). The arcR and queA genes appeared slightly more strongly upregulated in arginine restriction in S. gordonii $\triangle \operatorname{argR}$ and $\Delta a h r C$ mutants than the wild-type (Figure 4). The SGO_RS07910-07925 operon and manLM were weakly downregulated in no arginine in $S$. gordonii $\Delta \operatorname{argR}$ and $\Delta a h r C$. This pattern of gene expression was more similar to S. gordonii DL1 than to S. gordonii $\Delta$ arcR. By contrast, the pattern of arginine-dependent gene regulation in S. gordonii $\Delta \arg R$ and $\Delta a h r C$ for the S11 peptidase gene, SGO_RS07655 and SGO_RS04295 hypothetical protein-encoding genes and $\operatorname{argGH}$ was more similar to $S$. gordonii $\Delta \operatorname{arcR}$ than to the wild-type. SGO_RS09090 Clp protease ATP-binding protein locus was upregulated in no arginine to similar extents in all S. gordonii strains. Overall, these data demonstrate that disruption of $\operatorname{arcR}$ leads to several unique changes in gene expression that are not seen in S. gordonii $\Delta \operatorname{argR}$ or $\Delta a h r C$.

\section{5 | Role of SGO_RS04150 in biofilm formation}

Of the differentially regulated genes, SGO_RS04150 (SGO_0846) was of particular interest because it was strongly regulated in $\mathrm{S}$. gordonii $\Delta \operatorname{arcR}$. This gene is annotated as encoding a cell wall protein in the $\mathrm{NCBI}$ database. Using SignalP, ${ }^{31}$ we identified a putative $\mathrm{N}$-terminal secretion signal. However, using PSORTb ${ }^{32}$ we were unable to detect an LPXTG cell wall anchor or a lipoprotein motif and it is possible that the protein is secreted from the cell surface. To confirm that this 
gene is regulated by ArcR, we assessed expression levels by RT-qPCR under arginine-replete or no arginine conditions (Figure 5A). Under high arginine, expression was significantly increased 17 -fold in S. gordonii $\Delta \operatorname{arcR}$ compared with wild-type. In line with the microarray data (Figure 4), expression was slightly elevated in no arginine in S. gordonii DL1 and slightly decreased in S. gordonii $\Delta \operatorname{arcR}$. In view of the strong
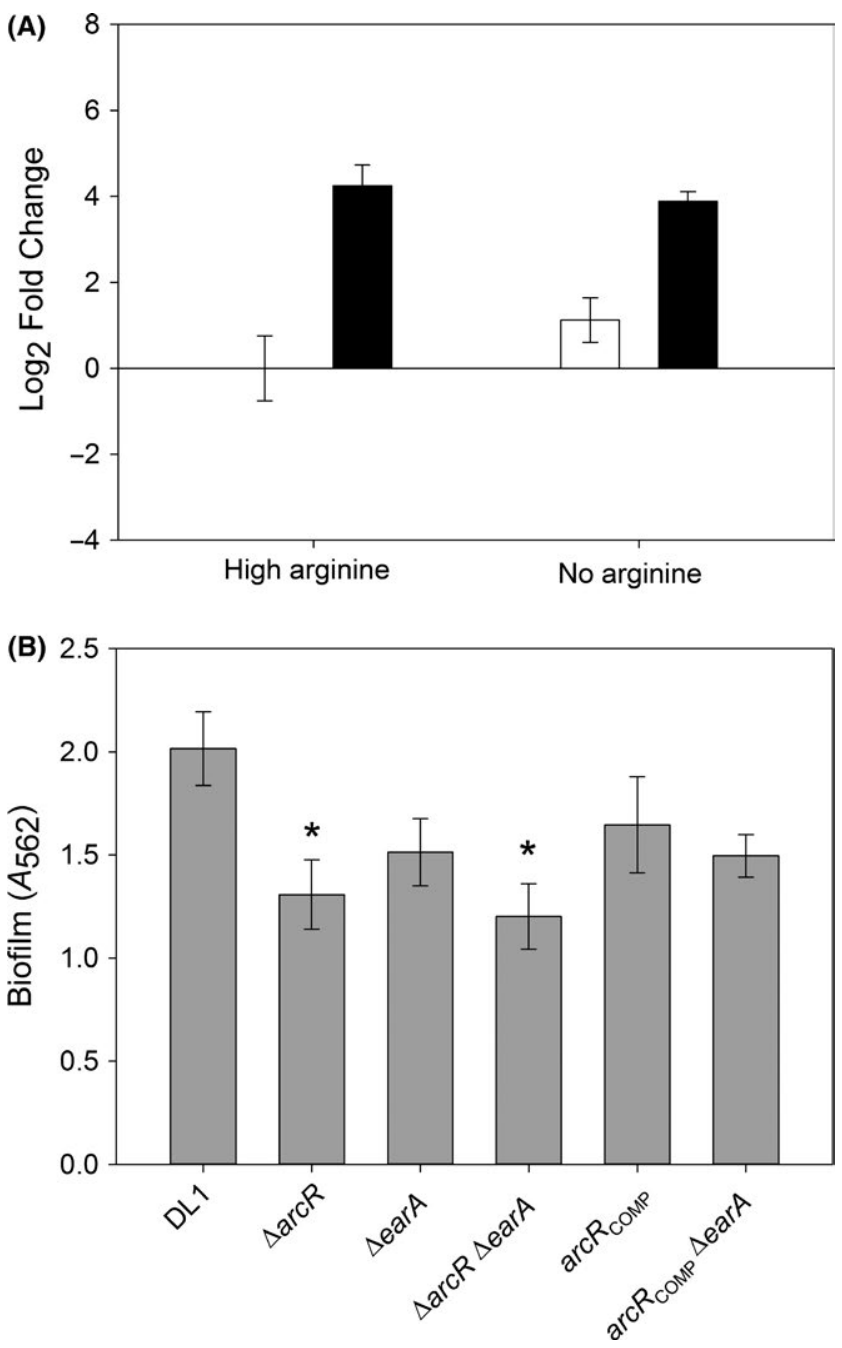

FIGURE 5 Expression of earA and impact of earA gene disruption on biofilm formation by Streptococcus gordonii. (A) RT-qPCR analysis of earA expression in S. gordonii DL1 and $\Delta \operatorname{arcR}$ under high and no arginine. Cells were grown in FMC medium, harvested and resuspended in FMC without arginine or arginine-replete FMC. Following incubation for $30 \mathrm{~min}$, gene expression was measured by RT-qPCR and is expressed as $\log _{2}$-fold change values. White bars represent the earA expression in S. gordonii DL1 and black bars represent earA expression in S. gordonii $\Delta$ arcR. Bars represent means of three independent biological replicates, and standard error is shown. (B) Impact of earA gene disruption on biofilm formation. Biofilms were grown in TYEG medium aerobically for $18 \mathrm{~h}$, and quantified by measuring absorbance of crystal violet-stained cells at $562 \mathrm{~nm}$. Bars represent means of three independent biological replicates, and error bars show standard error. Significant differences between mutants and wild-type are indicated by an asterisk. Disruption of earA did not significantly reduce biofilm formation in wild-type or $\triangle \operatorname{arcR}$ genetic backgrounds regulation by ArcR, we have termed the SGO_RS04150 gene "earA", encoding "Extracellular ArcR-Regulated protein A". To assess the role of earA in S. gordonii biofilm formation, the gene was disrupted in the wild-type and the $\triangle \operatorname{arcR}$ background. Using crystal violet staining, biofilm formation by $S$. gordonii $\triangle$ earA was slightly reduced compared with the wild-type (Figure 5B) but the difference was not significant. Similarly, knockout of earA in the $\Delta \operatorname{arcR}$ or $\operatorname{arc} R_{\text {Comp }}$ backgrounds did not significantly reduce biofilm formation. Therefore, it appears that dysregulation of earA expression is not the major cause of the biofilm defect observed in S. gordonii $\triangle \operatorname{arcR}$.

\section{4 | DISCUSSION}

Our studies identified ArcR as a key determinant of biofilm formation in S. gordonii. As no arginine metabolic gene disruptions affected biofilm formation, it appears that ArcR may be acting through a mechanism that is independent of regulation of arginine metabolism. Interestingly, a different ArgR family regulator, EF0676, the orthologue of ArgR, was identified in a transposon mutant screen for biofilm-associated genes in Enterococcus faecalis. ${ }^{33}$ Subsequently, the homologue of AhrC, EF0983, was also implicated in biofilm formation as strains carrying a transposon insertion were impaired in the development of biofilms in vitro and in biofilm infection models of infective endocarditis and catheter-associated urinary tract infection. ${ }^{34}$ The $E$. faecalis genome also contains two genes encoding orthologues of $S$. gordonii ArcR that have been named $\operatorname{argR2}$ and $\operatorname{argR} 1 .{ }^{35}$ However, to the best of our knowledge neither of these, or any other orthologues of $S$. gordonii $\operatorname{arcR}$, have yet been associated with biofilm formation phenotypes.

ArgR-family regulators form hexameric complexes that are activated by the binding of arginine, and bind to regulatory regions termed ARG box elements in the upstream region of target genes. ${ }^{36}$ It is not clear why streptococci and related bacteria produce multiple ArgR family homologues. In particular, the function of ArgR and $\mathrm{AhrC}$ is often very similar, and knocking out one or other gene is sufficient to abrogate arginine-dependent regulation of target genes. ${ }^{19}$ It has been postulated that these may form a heterohexameric complex or that they may co-ordinate gene expression by crossregulating one another. ${ }^{37}$ Transcriptome analysis in Lactococcus lactis or Streptococcus pneumoniae has identified minor differences between the ArgR (termed ArgR1 in S. pneumoniae) and AhrC regulons, indicating that heterohexameric complexes containing both ArgR and AhrC are not essential for gene regulation. ${ }^{38,39}$ By contrast, direct comparison between gene expression in S. gordonii $\Delta \arg R$ and $\Delta a h r C$ here did not identify any significant differences between the strains in either high or no arginine. Nearly $20 \%$ of the probes failed to reach the quality control thresholds and it is possible that some differences between the ArgR and AhrC regulons were missed in our analysis. Nevertheless, it appears that ArgR and AhrC have very similar functions in S. gordonii.

In a previous microarray analysis of $S$. gordonii DL1, > 450 genes were shown to be significantly regulated following a shift from high 
to no arginine. ${ }^{19}$ Streptococcus gordonii has the capacity to biosynthesize arginine, but does not grow aerobically in media lacking arginine. ${ }^{21}$ Therefore, it is possible that some of this gene regulation was an adaptation to stress rather than a specific response to the lack of arginine. In growth experiments using FMC medium, S. gordonii $\Delta \arg R, \Delta a h r C$ and $\triangle \operatorname{arcR}$ were similarly impaired in aerobic growth in the absence of arginine (data not shown). Therefore, differences in gene regulation between $S$. gordonii DL1 and the mutant strains are likely to reflect the function of the regulators rather than simply stress imposed by the lack of arginine and the subsequent growth arrest. Many of the genes that were regulated in S. gordonii DL1 following a shift from high to no arginine, including arginine and histidine biosynthesis operons, were upregulated specifically in high arginine in S. gordonii $\Delta \operatorname{argR}$ or $\Delta a h r C$ compared with wild-type (Table S4). This pattern of regulation is consistent with $\mathrm{ArgR} / \mathrm{AhrC}$ acting as repressors in arginine-bound form. Using the transcription factor binding site prediction program PePPER ${ }^{40}$ to search for L. lactis ARG box elements throughout the $S$. gordonii genome, six ARG boxes were identified upstream of genes that were upregulated in S. gordonii $\triangle \operatorname{argR}$ or $\Delta a h r C$. These were upstream of $\arg R, \arg C$ (two elements), pyrR, acnA and SGO_RS08115. Of these, $\operatorname{argR}$ encodes the ArgR regulator, $\arg C$ is the first gene in the $\arg C J B D$ arginine biosynthesis operon and pyrR is the first gene in the $\operatorname{pyr} \mathrm{A}_{a} A_{b}$ arginine biosynthesis operon. On the other hand, acnA, encoding aconitate hydratase and SGO_RS08115, encoding phosphoenolpyruvate carboxykinase, are involved in the citric acid cycle, indicating that arginine sensing is linked to central metabolism in S. gordonii. Although ArgR/AhrC are thought to act primarily as repressors of transcription, it is possible that they may also be activators in some conditions. The above screen identified one ARG box element upstream of $s c a C$, the first gene in the scaCBA operon encoding an ATP-binding cassette manganese transporter, ${ }^{41}$ that was downregulated in S. gordonii $\Delta \operatorname{argR}$ and $\Delta a h r C$ under high or low arginine. However, $s c a B$ was not regulated and $s c a A$ was only regulated in $S$. gordonii $\Delta \arg R$ under high arginine. Therefore, it is not clear what impact arginine-sensing has on the expression or function of the transporter as a whole. It is likely that there are many other ARG box elements upstream of ArgR/AhrC-regulated genes in S. gordonii. In Escherichia coli, 62 ArgR binding regions have been identified by chromatin immunoprecipitation (ChIP-Seq). ${ }^{36,42}$ Measurement of ArgR/AhrC interactions with DNA in promoter regions by ChIP-Seq, electrophoretic mobility shift or DNase I footprinting would be required to determine which genes are directly regulated by $\mathrm{ArgR} / \mathrm{AhrC}$ in S. gordonii.

The target DNA binding sequence for $S$. gordonii ArcR has been investigated by DNase I footprinting, and shown to consist of a 27-bp region with little identity to ARG box motifs. ${ }^{43}$ ArcR has previously been shown to regulate the expression of the $\operatorname{arc} A B C$ operon, queA and $\operatorname{argGH} .{ }^{19,44,45}$ The microarray analysis identified queA and $\arg G H$ as genes that were upregulated in $S$. gordonii $\triangle \operatorname{arcR}$ compared with wild-type under high arginine. Interestingly, however, $\operatorname{arcA}, \operatorname{arcB}$ and $\operatorname{arcC}$ were not differentially expressed between S. gordonii DL1 and $\triangle \operatorname{arcR}$ under high or no arginine by microarray analysis. All three genes were upregulated approximately three- to four-fold in high arginine compared with no arginine in both strains. It is well-established that disruption of the $\operatorname{arc} R$ gene abrogates arginine-dependent activation of $\operatorname{arcABC}$ expression ${ }^{19,45}$ and it is therefore not clear why this was not seen in the microarray data. However, using RT-qPCR, which has a higher dynamic range than microarrays, ${ }^{46}$ it was found that expression of $\operatorname{arc} A$ and $\operatorname{arcB}$ was significantly lower in S. gordonii $\triangle \operatorname{arcR}$ than the wild-type under high arginine, in line with previous observations (data not shown).

In addition to known genes, ArcR was shown to have a major impact on the regulation of the phosphoenolpyruvate:sugar phosphotransferase system (PTS) components. Oral streptococci use many different carbohydrates for growth and PTSs are the primary mechanism for sugar uptake. ${ }^{47}$ Using proteomic analysis, it was found that PTSs are a common target for gene regulation in S. gordonii following interspecies interactions with P. gingivalis and F. nucleatum. ${ }^{48}$ In addition, the PTS operon fruRBA was upregulated following adhesion of $S$. gordonii to C. albicans. ${ }^{20}$ This operon was previously shown to have a key role in biofilm formation. ${ }^{49}$ Although fruRBA was not regulated by ArcR, another PTS (levDEFG) was strongly (7- to 12-fold) upregulated in $S$. gordonii $\triangle \operatorname{arcR}$ under high arginine, indicating that $A r c R$ represses expression of this operon when arginine is available. The genes leVDEFG encode enzyme IA, IIA, IIB and IIC components of a PTS that primarily transports fructose. ${ }^{50}$ It is not yet known whether this system is required for the development of biofilms by S. gordonii. Similarly, the PTS enzyme IIAB and IIC components encoded by manLM have been assessed for their roles in sugar uptake and found to transport glucose, galactose and mannose, but have not been assessed for biofilmassociated functions. ${ }^{50}$ Three additional PTSs have been shown to be important for biofilm formation by S. gordonii. ${ }^{51}$ Of these, the bfb locus encoding a cellobiose PTS has previously been shown to be regulated by arginine and by coaggregation with A. oris. ${ }^{19,21}$ Although the bfb genes were not significantly regulated in $S$. gordonii $\Delta \operatorname{arcR}$ compared with wild-type in our microarray analyses, there was a trend towards upregulation under high arginine. Therefore, we assessed the expression of $b f b C$ and bfbF in S. gordonii DL1 and $\triangle a r c R$ by RT-qPCR. Both genes were approximately four-fold increased in $S$. gordonii $\Delta \operatorname{arcR}$ compared with wild-type under high arginine, and the difference was statistically significant $(P<.05)$ in the case of $b f b C$. Therefore, it is possible that dysregulation of the $b f b$ locus in the absence of ArcR may be partly responsible for the observed biofilm defects of $S$. gordonii $\Delta \operatorname{arcR}$.

In addition to PTSs, disruption of $\operatorname{arcR}$ also led to a change in the regulation of an apparent four-gene operon containing a magnesium transporter, CAAX amino protease, methyltransferase and peptide chain release factor 3 . These genes were only weakly regulated by arginine in S. gordonii DL1, but were more strongly downregulated by a shift to no arginine in S. gordonii $\Delta \operatorname{arcR}$. It is not clear why these genes are linked to arginine sensing in S. gordonii. However, it is noteworthy that this operon is immediately upstream of acnA, which contains an ARG box element in the promoter region. The gene encoding a protease was one of two CAAX protease-encoding genes that were regulated by ArcR. The function of these proteins is not fully understood, but some of them play a role in self-immunity against bacteriocins. $^{52}$ Oral streptococci possess many paralogues of genes encoding 
CAAX amino proteases: S. gordonii has 14, whereas S. sanguinis has 21 paralogues, more than any other species that has been analyzed to date. ${ }^{52}$ One of these proteases in S. gordonii (BfrH) and two in S. sanguinis ( $\mathrm{BfrH} 1$ and $\mathrm{BfrH} 2$ ) are regulated by biofilm-associated $\mathrm{BfrAB}$ two-component systems and it has been speculated that these may play important roles in processing proteins that are secreted to promote biofilm development. ${ }^{53}$ The ClpP protease complex may also be involved in controlling the quality and integrity of secreted proteins involved in biofilms. Hence, an ATP binding protein associated with the ClpP complex was upregulated in S. gordonii $\Delta \operatorname{arcR}$ compared with wild-type under high arginine. The ClpP complex consists of ClpP protease and a number of ATPases that together are required for biofilm formation in Streptococcus mutans. ${ }^{54}$ Disruption of the $\operatorname{arcR}$ gene also led to a change in expression of the competence protein CoiA. In S. pneumoniae CoiA is involved in genetic recombination and is only transiently expressed during competence. It is possible that disruption of $\operatorname{arcR}$ led to the initiation of competence development, ${ }^{55}$ although it is important to note that other competence genes were not differentially regulated between S. gordonii DL1 and $\Delta \operatorname{arcR}$. The gene encoding carboxylate-amine ligase is immediately upstream of purE involved in purine biosynthesis, and may even be part of the same operon. The purE gene was downregulated in S. gordonii $\triangle \arg R$ and $\triangle a h r C$ compared with wild-type under high and no arginine. Therefore, it appears that all three ArgR family regulators may co-ordinate to control this locus, as they do for the regulation of $\operatorname{argGH}$. Finally, peptidase S11 is a D-alanyl-D-alanine carboxypeptidase, involved in peptidoglycan biosynthesis. It is not clear whether this or any of the hypothetical proteins that were regulated by ArcR could be involved in biofilm formation.

The strongest regulation in S. gordonii $\Delta \operatorname{arcR}$ was a gene of unknown function, which we have termed earA. This gene was upregulated in S. gordonii following a shift to no arginine. However, in S. gordonii $\triangle \operatorname{arcR}$ expression of earA was strongly ( $>100$-fold in the microarray analysis) upregulated under high arginine. The function of EarA is unknown. It is predicted to have a cysteine-rich secretory protein (SCP)-like extracellular protein domain; the function of these domains is not well-understood. ${ }^{56}$ It was possible that the dramatic increase in expression of earA in S. gordonii $\triangle a r c R$ may be associated with the observed biofilm defect. However, disruption of the earA gene in S. gordonii DL1, $\Delta \operatorname{arcR}$ or $\operatorname{arcR}_{\text {Comp }}$ did not affect biofilm formation compared with the respective parent strain, indicating that EarA is not a major contributor to biofilm development.

In conclusion, we have identified ArcR as a key gene in biofilm formation by $S$. gordonii. The mechanism by which ArcR affects biofilm growth or stability remains unclear, but it is possible that changes in the co-ordination of PTS expression may be at least partly responsible. Further work is required to establish whether arginine is the only stimulus for ArcR, and to determine how arginine itself modulates $S$. gordonii biofilm development through activation of the ArcR regulatory pathway. In addition, assessment of the role of ArcR in the early stages of biofilm formation, or in biofilm formation under fluid flow, may help to shed light on the specific function of ArcR in biofilm development by S. gordonii.

\section{ACKNOWLEDGEMENTS}

We are very grateful to Lesley Old for expert technical assistance. We thank Lorraine Wallace, Functional Genomics, Proteomics and Metabolomics Facility, University of Birmingham, for conducting the microarray hybridization experiments. We also acknowledge the support of Matthew Bashton, Bioinformatics Core Facility, Newcastle University, for preliminary analysis of the microarray data. The work was supported by a Jeffcock and Luccock Newcastle University PhD studentship awarded to J.C.R. There are no conflicts of interest to declare.

\section{ORCID}

N.S. Jakubovics (iD http://orcid.org/0000-0001-6667-0515

\section{REFERENCES}

1. Jakubovics NS. Intermicrobial interactions as a driver for community composition and stratification of oral biofilms. J Mol Biol. 2015;427:3662-3675

2. Nobbs AH, Jenkinson HF, Jakubovics NS. Stick to your gums: mechanisms of oral microbial adherence. J Dent Res. 2011;90:1271-1278.

3. Jakubovics NS, Yassin SA, Rickard AH. Community interactions of oral streptococci. Adv Appl Microbiol. 2014;87:43-110.

4. Kreth J, Merritt J, Qi F. Bacterial and host interactions of oral streptococci. DNA Cell Biol. 2009;28:397-403.

5. Zhou P, Liu J, Li X, Takahashi Y, Qi F. The sialic acid binding protein, $\mathrm{Hsa}$, in Streptococcus gordonii DL1 also mediates intergeneric coaggregation with Veillonella species. PLoS ONE. 2015;10:e0143898.

6. Back CR, Douglas SK, Emerson JE, Nobbs AH, Jenkinson HF. Streptococcus gordonii DL1 adhesin SspB V-region mediates coaggregation via receptor polysaccharide of Actinomyces oris T14V. Mol Oral Microbiol. 2015;30:411-424.

7. McNab R, Forbes H, Handley PS, Loach DM, Tannock GW, Jenkinson HF. Cell wall-anchored CshA polypeptide (259 kilodaltons) in Streptococcus gordonii forms surface fibrils that confer hydrophobic and adhesive properties. J Bacteriol. 1999;181:3087-3095.

8. Bor DH, Woolhandler S, Nardin R, Brusch J, Himmelstein DU. Infective endocarditis in the U.S., 1998-2009: a nationwide study. PLoS ONE. 2013;8:e60033.

9. Huang $X$, Schulte RM, Burne RA, Nascimento MM. Characterization of the arginolytic microflora provides insights into $\mathrm{pH}$ homeostasis in human oral biofilms. Caries Res. 2015;49:165-176.

10. Nascimento MM, Browngardt C, Xiaohui X, Klepac-Ceraj V, Paster BJ, Burne RA. The effect of arginine on oral biofilm communities. Mol Oral Microbiol. 2014;29:45-54.

11. Nascimento MM, Liu $\mathrm{Y}$, Kalra R, et al. Oral arginine metabolism may decrease the risk for dental caries in children. J Dent Res. 2013;92:604-608.

12. Ho MH, Lamont RJ, Xie H. Identification of Streptococcus cristatus peptides that repress expression of virulence genes in Porphyromonas gingivalis. Sci Rep. 2017;7:1413.

13. Sakanaka A, Kuboniwa M, Takeuchi $\mathrm{H}$, Hashino E, Amano A. Arginineornithine antiporter ArcD controls arginine metabolism and interspecies biofilm development of Streptococcus gordonii. J Biol Chem. 2015;290:21185-21198.

14. Edwards AM, Grossman TJ, Rudney JD. Association of a highmolecular weight arginine-binding protein of Fusobacterium nucleatum ATCC 10953 with adhesion to secretory immunoglobulin A and coaggregation with Streptococcus cristatus. Oral Microbiol Immunol. 2007;22:217-224. 
15. Kaplan CW, Lux R, Haake SK, Shi W. The Fusobacterium nucleatum outer membrane protein RadD is an arginine-inhibitable adhesin required for inter-species adherence and the structured architecture of multispecies biofilm. Mol Microbiol. 2009;71:35-47.

16. Kolderman E, Bettampadi D, Samarian D, et al. L-Arginine destabilizes oral multi-species biofilm communities developed in human saliva. PLoS ONE. 2015;10:e0121835.

17. Tada A, Nakayama-Imaohji $\mathrm{H}$, Yamasaki $\mathrm{H}$, et al. Cleansing effect of acidic L-arginine on human oral biofilm. BMC Oral Health. 2016;16:40.

18. Huang $X$, Zhang $K$, Deng $M$, et al. Effect of arginine on the growth and biofilm formation of oral bacteria. Arch Oral Biol. 2017;82:256-262.

19. Jakubovics NS, Robinson JC, Samarian DS, et al. Critical roles of arginine in growth and biofilm development by Streptococcus gordonii. Mol Microbiol. 2015;97:281-300.

20. Jesionowski AM, Mansfield JM, Brittan JL, Jenkinson HF, Vickerman MM. Transcriptome analysis of Streptococcus gordonii Challis DL1 indicates a role for the biofilm-associated fruRBA operon in response to Candida albicans. Mol Oral Microbiol. 2016;31:314-328.

21. Jakubovics NS, Gill SR, lobst SE, Vickerman MM, Kolenbrander PE. Regulation of gene expression in a mixed-genus community: stabilized arginine biosynthesis in Streptococcus gordonii by coaggregation with Actinomyces naeslundii. J Bacteriol. 2008;190:3646-3657.

22. Sambrook J, Russell DW. Molecular Cloning: a Laboratory Manual, 3rd edn. Cold Spring Harbor, N.Y.: Cold Spring Harbor Laboratory Press; 2001.

23. Hansen MC, Palmer RJ Jr, Udsen C, White DC, Molin S. Assessment of GFP fluorescence in cells of Streptococcus gordonii under conditions of low $\mathrm{pH}$ and low oxygen concentration. Microbiology. 2001;147:1383-1391.

24. Egland PG, Palmer RJ Jr, Kolenbrander PE. Interspecies communication in Streptococcus gordonii-Veillonella atypica biofilms: signaling in flow conditions requires juxtaposition. Proc Natl Acad Sci U S A. 2004;101:16917-16922.

25. Shields RC, Mokhtar N, Ford M, et al. Efficacy of a marine bacterial nuclease against biofilm forming microorganisms isolated from chronic rhinosinusitis. PLoS ONE. 2013;8:e55339.

26. Benjamini Y, Hochberg Y. Controlling the false discovery rate - a practical and powerful approach to multiple testing. J R Stat Soc Ser BMethodol. 1995;57:289-300.

27. Del Carratore F, Jankevics A, Eisinga R, Heskes T, Hong F, Breitling R. RankProd 2.0: a refactored Bioconductor package for detecting differentially expressed features in molecular profiling datasets. Bioinformatics. 2017;33:2774-2775.

28. R Core Team. R: A language and environment for statistical computing. 2017; https://www.R-project.org/. Accessed July 1, 2017.

29. Gu Z, Eils R, Schlesner M. Complex heatmaps reveal patterns and correlations in multidimensional genomic data. Bioinformatics. 2016;32:2847-2849.

30. Manimaran S, Selby HM, Okrah K, et al. BatchQC: interactive software for evaluating sample and batch effects in genomic data. Bioinformatics. 2016;32:3836-3838.

31. Petersen TN, Brunak S, vonHeijne G, Nielsen H. SignalP 4.0: discriminating signal peptides from transmembrane regions. Nat Methods. 2011;8:785-786.

32. Yu NY, Wagner JR, Laird MR, et al. PSORTb 3.0: improved protein subcellular localization prediction with refined localization subcategories and predictive capabilities for all prokaryotes. Bioinformatics. 2010;26:1608-1615.

33. Kristich CJ, Nguyen VT, Le T, Barnes AM, Grindle S, Dunny GM. Development and use of an efficient system for random mariner transposon mutagenesis to identify novel genetic determinants of biofilm formation in the core Enterococcus faecalis genome. Appl Environ Microbiol. 2008;74:3377-3386.

34. Frank KL, Guiton PS, Barnes AM, et al. AhrC and Eep are biofilm infection-associated virulence factors in Enterococcus faecalis. Infect Immun. 2013;81:1696-1708.
35. Barcelona-Andres B, Marina A, Rubio V. Gene structure, organization, expression, and potential regulatory mechanisms of arginine catabolism in Enterococcus faecalis. J Bacteriol. 2002;184:6289-6300.

36. Cho S, Cho YB, Kang TJ, Kim SC, Palsson B, Cho BK. The architecture of ArgR-DNA complexes at the genome-scale in Escherichia coli. Nucleic Acids Res. 2015;43:3079-3088.

37. Larsen R, Kok J, Kuipers OP. Interaction between ArgR and AhrC controls regulation of arginine metabolism in Lactococcus lactis. J Biol Chem. 2005;280:19319-19330.

38. Kloosterman TG, Kuipers OP. Regulation of arginine acquisition and virulence gene expression in the human pathogen Streptococcus pneumoniae by transcription regulators ArgR1 and AhrC. J Biol Chem. 2011;286:44594-44605.

39. Larsen R, van Hijum SA, Martinussen J, Kuipers OP, Kok J. Transcriptome analysis of the Lactococcus lactis ArgR and AhrC reguIons. Appl Environ Microbiol. 2008;74:4768-4771.

40. De Jong A, Pietersma H, Cordes M, Kuipers OP, Kok J. PePPER: a webserver for prediction of prokaryote promoter elements and regulons. BMC Genom. 2012;13:299.

41. Jakubovics NS, Smith AW, Jenkinson HF. Expression of the virulencerelated Sca $\left(\mathrm{Mn}^{2+}\right)$ permease in Streptococcus gordonii is regulated by a diphtheria toxin metallorepressor-like protein ScaR. Mol Microbiol. 2000;38:140-153.

42. Cho BK, Federowicz S, Park YS, Zengler K, Palsson BO. Deciphering the transcriptional regulatory logic of amino acid metabolism. Nat Chem Biol. 2012;8:65-71.

43. Zeng L, Dong Y, Burne RA. Characterization of cis-acting sites controlling arginine deiminase gene expression in Streptococcus gordonii. J Bacteriol. 2006:188:941-949.

44. Liu Y, Dong Y, Chen YY, Burne RA. Environmental and growth phase regulation of the Streptococcus gordonii arginine deiminase genes. Appl Environ Microbiol. 2008;74:5023-5030.

45. Dong Y, Chen YY, Snyder JA, Burne RA. Isolation and molecular analysis of the gene cluster for the arginine deiminase system from Streptococcus gordonii DL1. Appl Environ Microbiol. 2002;68:5549-5553.

46. Jozefczuk J, Adjaye J. Quantitative real-time PCR-based analysis of gene expression. Methods Enzymol. 2011;500:99-109.

47. Moye ZD, Zeng L, Burne RA. Fueling the caries process: carbohydrate metabolism and gene regulation by Streptococcus mutans. J Oral Microbiol. 2014;6.doi: 10.3402/jom.v3406.24878.

48. Hendrickson EL, Wang TS, Dickinson BC, et al. Proteomics of Streptococcus gordonii within a model developing oral microbial community. BMC Microbiol. 2012;12:211.

49. Loo CY, Mitrakul K, Voss IB, Hughes CV, Ganeshkumar N. Involvement of an inducible fructose phosphotransferase operon in Streptococcus gordonii biofilm formation. J Bacteriol. 2003;185:6241-6254.

50. Tong H, Zeng L, Burne RA. The EllABMan phosphotransferase system permease regulates carbohydrate catabolite repression in Streptococcus gordonii. Appl Environ Microbiol. 2011;77:1957-1965.

51. Kilic AO, Tao L, Zhang Y, Lei Y, Khammanivong A, Herzberg MC. Involvement of Streptococcus gordonii $\beta$-glucoside metabolism systems in adhesion, biofilm formation, and in vivo gene expression. $J$ Bacteriol. 2004;186:4246-4253.

52. Pei J, Mitchell DA, Dixon JE, Grishin NV. Expansion of type II CAAX proteases reveals evolutionary origin of $\gamma$-secretase subunit APH-1. J Mol Biol. 2011;410:18-26.

53. Zhang $\mathrm{Y}$, Whiteley $\mathrm{M}$, Kreth J, et al. The two-component system BfrAB regulates expression of $A B C$ transporters in Streptococcus gordonii and Streptococcus sanguinis. Microbiology. 2009;155: 165-173.

54. Zhang JQ, Hou XH, Song XY, Ma XB, Zhao YX, Zhang SY. ClpP affects biofilm formation of Streptococcus mutans differently in the presence of cariogenic carbohydrates through regulating gtfBC and ftf. Curr Microbiol. 2015;70:716-723. 
55. Desai BV, Morrison DA. Transformation in Streptococcus pneumoniae: formation of eclipse complex in a coiA mutant implicates CoiA in genetic recombination. Mol Microbiol. 2007;63:1107-1117.

56. Yeats C, Bentley S, Bateman A. New knowledge from old: in silico discovery of novel protein domains in Streptomyces coelicolor. BMC Microbiol. 2003;3:3.

\section{SUPPORTING INFORMATION}

Additional Supporting Information may be found online in the supporting information tab for this article.
How to cite this article: Robinson JC, Rostami N, Casement J, Vollmer W, Rickard AH, Jakubovics NS. ArcR modulates biofilm formation in the dental plaque colonizer Streptococcus gordonii. Mol Oral Microbiol. 2018;33:143-154. https://doi.org/10.1111/ omi.12207 